\title{
Flavour-singlet pseudoscalar and scalar mesons
}

\author{
UKQCD Collaboration, C. Michael, M. S. Foster* and C. McNeile
}

Theoretical Physics Division, Dept. of Mathematical Sciences, University of Liverpool, Liverpool L69 $3 \mathrm{BX}, \mathrm{UK}$

We measure correlations appropriate for favour-singlet mesons using dynamical quark configurations from UKQCD. Improved methods of evaluating the disconnected quark diagrams are presented. The scalar and pseudoscalar meson channels are explored, with special reference to the mixing of scalar mesons with scalar glueballs.

\section{INTRODUCTION}

To study flavour singlet mesons, we need to consider quark loops which are disconnected (often called hairpins), namely evaluate $\operatorname{Tr} \Gamma \mathrm{M}^{-1}$ where the sum (trace) is over all space (for zero momentum) at a given time value and all colours and spins. Here $M$ is the lattice fermion matrix, $\Gamma$ is a combination of the appropriate $\gamma$-matrix and a product of gauge links if a non-local operator is used for the meson.

Using a random volume source $\xi$ (where $\left\langle\xi^{*} \xi\right\rangle=1$ for the same colour, spin and spacetime component and zero otherwise) then solving $M \phi=\xi$, one can evaluate unbiased estimates of the propagator $M_{x y}^{-1}$ from $\left\langle\xi_{y}^{*} \phi_{x}\right\rangle$ where the average is over the $N_{S}$ samples of the stochastic source. The drawback of this approach is that the variance on these estimates can be very large, so that typically hundreds of samples are needed. Here we present a method which succeeds in reducing this variance substantially at rather small computational expense.

The variance reduction is based on expressing the fermion matrix $M$ as

$M=C+D=C\left(1+C^{-1} D\right)=\left(1+D C^{-1}\right) C(1)$

where $C$ is easy to invert, for example the SWclover term which is local in space. Then we have the exact identity

$$
\begin{aligned}
M^{-1} & =C^{-1}-C^{-1} D C^{-1}+\ldots+\left(-C^{-1} D\right)^{m} C^{-1} \\
& +\left(-C^{-1} D\right)^{n_{1}} M^{-1}\left(-D C^{-1}\right)^{n_{2}}
\end{aligned}
$$

*present address: MVC, Manchester Computing, Manchester M13 9PL with $n_{1}+n_{2}=n=m+1$.

Using the stochastic estimate for $M^{-1}$ on the rhs will reduce the variance of the estimate given by the lhs since the terms not involving $M^{-1}$ can be evaluated either exactly (for example terms with odd powers of $D$ vanish in the evaluation of a local trace) or as a subsidiary stochastic calculation with more samples since no inversion is required.

A special case of this $\left(n_{1}=n_{2}=2\right)$ with Wilson fermions (for which $C=1$ and the terms with up to 3 powers of $D$ vanish for $\operatorname{TrM}^{-1}$ ) was used by the bermion group [1] previously.

Using larger values of $n_{1}$ and $n_{2}$ implies that the estimate of $M^{-1}$ is very non-local. To evaluate correlators between traces at $t_{1}$ and $t_{2}$, one must require that the samples of stochastic volume source used in the two cases are different so that there is no bias. We use $N_{S}=24$ stochastic samples and this condition is readily implemented. This number of samples was chosen to make the stochastic sampling error smaller (actually about $50 \%$ in the cases to be discussed next) than the intrinsic variance from one gauge configuration to another. The computational effort is equivalent to that in obtaining two conventional propagators (from two sources of all colour-spins)

We now apply this technique to $12^{3} 24$ lattices with $N_{f}=2$ at $\beta=5.2$ with $C_{S W}=1.76$ from UKQCD [2]. We use valence quark masses equal to the sea-quark mass determined by the $\kappa$-values as given in Table 1. In order to improve the statistics we measure the disconnected diagrams on configurations separated by less trajectories than for the connected correlators giving 253, 169 con- 
figurations at $\kappa=0.1395,0.1398$, respectively.

\section{PSEUDOSCALAR MESONS}

Since we are exploring $N_{f}=2$ we describe the flavour non-singlet $(I=1)$ pseudoscalar meson as $\pi$ and the flavour singlet $(I=0)$ as $\eta$. It is possible to apply our results to the physical case of 3 light quark flavours and the $\eta, \eta^{\prime}$ splitting as has been done from the quenched case [3], but we do not discuss this here.

We use local and fuzzed operators matching the calculation of the connected meson propagators [2]. Then including our results for the hairpin diagrams with the appropriate sign, we can fit the flavour singlet correlator to the $\eta$ mass and excited states. Even though, as discussed above, we have measured the disconnected quark loop correlator from every time slice to every other with a stochastic error which is effectively negligible, the signal is very noisy. This arises since the disconnected part of the $\eta$ correlation has an error which does not decrease with increasing time separation $T$, unlike the connected correlator where the error is roughly constant as a percentage of the signal with increasing $t$. To illustrate this, we show the ratio of disconnected $D$ to connected component $C$ of the $\eta$ correlator in fig. 1 .

The line shows the expectation coming from one state $(\pi)$ contributing to $C$ and one state $(\eta)$ contributing to $C-D$ with a mass difference in lattice units of 0.16. There is some sign in our data of the impact of unitarity for matched valence and sea quarks: namely that $D / C$ approaches 1 from below at large $t$. Making a fit with this constraint to $D / C$, it is possible to estimate the $\eta-\pi$ mass difference and we see that it does increase as the sea quark mass is decreased. Assuming that the $\eta$ mass is constant as the quark mass is decreased, we would get an $\eta$ mass in the chiral limit of around $800 \mathrm{MeV}$, of course with an uncontrolled systematic error

Because the flavour singlet correlator becomes noisy so rapidly with increasing time separation, it is very valuable to use a more extended basis for the meson (eg. local and fuzzed operators) to help in extracting the ground state signal. The 2 state fit from $t=3$ to 9 is shown in Table 1 .

\begin{tabular}{lllll}
\hline$\kappa$ & $r_{0} / a$ & $m_{\pi} a$ & $m_{\pi} / m_{\rho}$ & $m_{\eta} a$ \\
\hline 0.1395 & 3.44 & $0.558(8)$ & 0.71 & $0.65(6)$ \\
0.1398 & 3.65 & $0.476(16)$ & 0.67 & $0.62(15)$ \\
\hline
\end{tabular}

Table 1

The zero-momentum correlator has contributions increasing like $L^{3}$ for spatial volume $L^{3}$ whereas the noise increases like $L^{6}$. Thus we find a better signal to noise ratio for smaller volumes hence we present here only the results for $L=12$.

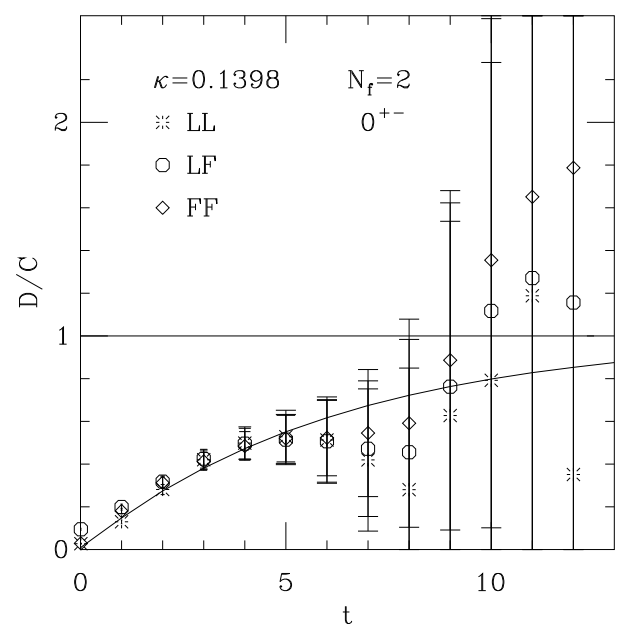

Figure 1. Disconnected to connected ratio.

\section{SCALAR MESONS}

For scalar mesons, we have the interesting situation that the $q \bar{q}$ mesons and the glueball can mix. Within the quenched approximation, it is possible to estimate this mixing 肺. We make a preliminary study in the quenched approximation at $\beta=5.7$ of 100 configurations on $12^{3}$ 24 lattices with $\mathrm{SW}$-clover valence quarks having $\kappa=0.14077$ with $C_{S W}=1.57$ (here $a \approx 1 \mathrm{GeV}$ and the quark mass is close to strange). We use 4 scalar meson operators (i) closed Wilson loops (glueball operators) of two different sizes (Tepersmeared) and (ii) $q \bar{q}$ operators which are local and separated by fuzzed links. Using the glueball mass of 0.97(4) from ref. [⿴囗十 and scalar $q \bar{q}$ mass of 1.48(15) from fitting the connected correlations, we are able to fit the disconnected correlation and the hairpin-Wilson loop correlation from $t$-values of 1 to 3 with a mixing given by $E a=0.4$ assuming $N_{f}=2$. This fit (see fig. 2) assumes only 


\begin{tabular}{llll}
\hline$\kappa$ & $m_{G B} a$ & $m_{F S} a$ & $m_{N S} a$ \\
\hline 0.1395 & $0.47(6)$ & $0.44(3)$ & $1.28(7)$ \\
0.1398 & $0.50(10)$ & $0.52(8)$ & $1.03(7)$ \\
\hline Table 2 & & &
\end{tabular}

ground state contributions so that the systematic error on $E$ from this assumption is hard to estimate. The mixing estimated by ref [- 1 ] is similar in magnitude at these lattice parameters $(E \approx 0.3$ $\mathrm{GeV}$ ) but they claim that on extrapolation to the continuum limit a much smaller value is obtained. Here we are using clover improvement so order $a$ effects are suppressed. If our quenched mixing strength were be to applied to the scalar mass matrix, it results in a downward shift for $N_{f}=2$ of the lattice glueball mass by $20 \%$.

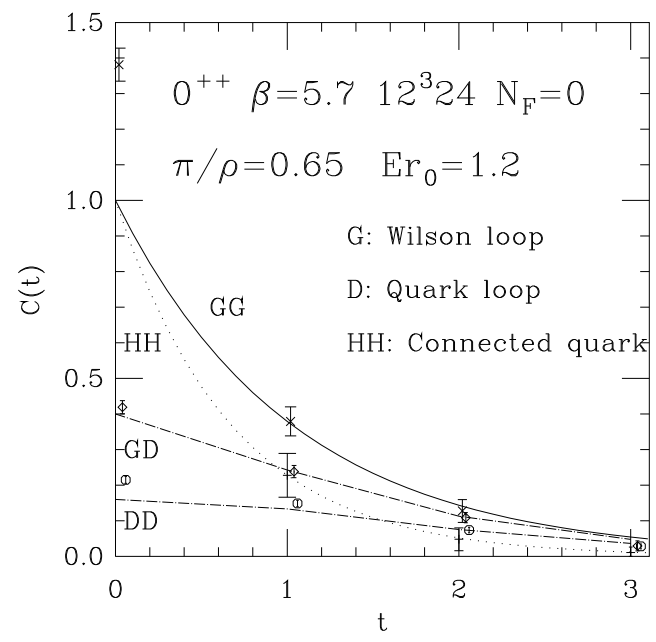

Figure 2. Quenched scalar correlations.

We can now measure directly the scalar spectrum for $N_{f}=2$ to explore this. We again use both glueball and $q \bar{q}$ operators and fit with 2 states the $4 \times 4$ matrix of correlations for $t$ from 2 to 10: the results is shown as $m_{F S} a$ in Table 2. We find that the mass obtained from fitting only the glueball correlations $\left(m_{G B}\right)$ is consistent with the full fit, as it should be. Moreover, we see a surprisingly low scalar mass - as emphasised in fig. 3 which compares with quenched results and the SESAM $N_{f}=2$ values [5.

We do expect a relatively light flavour-singlet scalar mass because of mixing effects as described above which would reduce the mass by $20 \%$. This could explain in part our low scalar mass but other explanations are also worth exploring. For example the order $a^{2}$ corrections might be anomalously large for our lattice implementation (e.g. twice as large as in the quenched Wilson case).

Another possible explanation of the light flavour-singlet scalar mass we find (comparable to our pion mass) would be a partial restoration of chiral symmetry in a finite volume. Our spatial size is $1.7 \mathrm{fm}$ and no evidence of finite size effects was seen in a study of flavour non-singlet correlators [2]. We have made a preliminary study of flavour singlet correlators on $16^{3}$ spatial lattices to check for finite size effects and, although the signal from the larger spatial volume is relatively noisier, we do see some evidence (at the $2 \sigma$ level) of a higher scalar mass on the larger spatial lattice.

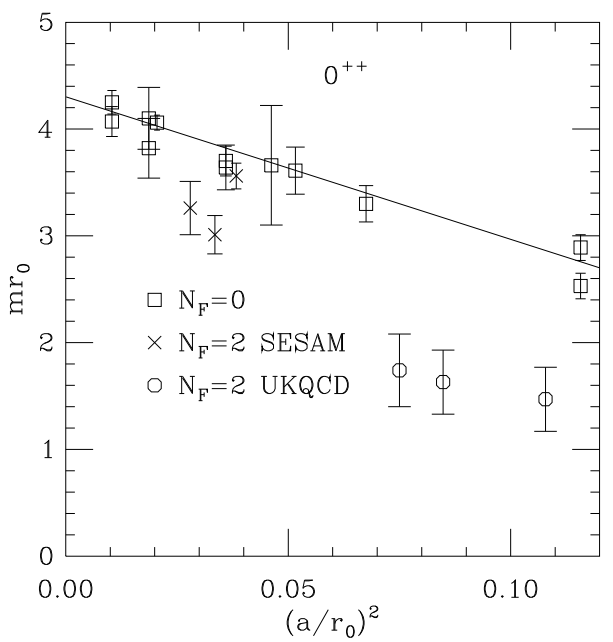

Figure 3. The scalar mass versus $a^{2}$.

\section{REFERENCES}

1. R Frezzotti,M Masetti and R Petronzio, Nucl. Phys. B480 (1996) 381.

2. UKQCD Collaboration, C. R. Allton et al., Phys. Rev. D60, 54012 (1999).

3. Y. Kuramashi et al., Phys. Rev. Lett. 72, 3348 (1994).

4. W. Lee and D. Weingarten, Nucl. Phys. Proc. Suppl. 63 (1998) 194; 73 (1999) 249.

5. G. Bali et al., Nucl. Phys. Proc. Suppl. 63 (1998) 209. 